\title{
Palabras clave apropiadas
}

Daniel Tena Parera, Universitat Autònoma de Barcelona; danieltenaparera@gmail.com

RESUMEN

Acertar con las palabras clave para la vinculación de un contenido con el continente que lo transporta y facilitar así su recuperación y uso es uno de los aspectos fundamentales que los metadatos de todo artículo científico deben cumplir. Entre los metadatos, las palabras clave no disponen todavía del suficiente interés para otorgarles una estrategia de uso como tampoco hay unas rutinas claras para su redacción. El presente texto pone de relieve la relevancia de las palabras clave, indica algunas pautas y evidencia el uso que se ha realizado hasta la fecha en la revista científica "grafica".

Palabras clave

Palabras clave; visualización; indexación

\section{ABSTRACT}

\section{Appropriated Keywords}

Getting the keywords right to link a content with the container that transports it and thus facilitate its retrieval and use is one of the fundamental aspects that the metadata of any paper must comply with. Among the metadata, the keywords are not yet of sufficient interest to give them a strategy for use, nor are there clear routines for their writing. This text highlights the relevance of keywords, indicates some guidelines and shows the use that has been made to date in the journal "graphic".

\section{Keywords}

Keywords; visualitation; indexing 
Uno de los elementos más relevantes de un artículo científico son los metadatos. A menudo, son olvidados por los autores, redactados de manera imprecisa o poco estratégica. Efectivamente, el título del artículo, su resumen y también las palabras clave son aspectos fundamentales para la visualización del contenido y consecuentemente, para la transferencia de conocimiento. En las líneas que siguen abordamos uno de estos elementos: las palabras clave utilizadas por "grafica" entre los años 2012 y 2020 . Y lo hacemos analizando las palabras clave utilizadas por los autores que han publicado artículos científicos y ensayos en gráfica. Esto nos va a permitir reflexionar sobre la paleta de palabras clave que podemos y debemos utilizar para potenciar nuestro trabajo.

En ser aceptado un artículo y ya cuando nos avisan para cerrarlo para ser publicado, suele ser el momento de las prisas, de la emoción, de los nervios. De repasar la galerada para informar a los gestores de la revista que se da el "adelante". En ese preciso momento, debemos asegurar que las palabras clave indicadas al principio del proceso de revisión, no han sido puestas en cuestión por el editor, por los revisores y determinar que son las que, finalmente, deben vincularse para siempre con el trabajo. Por lo general, los metadatos deben validarse en las correspondientes ventanas de la aplicación de edición. Y son los autores quienes deben acertar con esta validación.

Pero ¿Cuál es el problema real con las palabras clave? Desde la perspectiva de "grafica", nos parece que las palabras clave utilizadas en los artículos publicados no suelen servir para centrar su contenido ni alinearlo con otros contenidos, en esencia, semejantes. Así, si se utiliza una palabra clave como "Diseño" o "Diseño gráfico" puede parecer excesivamente genérico; mientras que si utilizamos neologismos, puede ser que no acertemos con su relevancia.

En definitiva: ¿Cómo podremos acertar con las palabras clave apropiadas en el caso de publicar en "grafica"?; ¿Cómo van a poder dotarse de mayor relevancia los artículos publicados en "gráfica" y sobresalir ante la gran cantidad de contenidos de diseño gráfico?

\section{Normas básicas para palabras clave}

Lo realmente relevante no es la visualización de grafica. Lo que realmente se debe trabajar es la visibilidad de cada uno de los artículos en los entornos digitales. En realidad, cuando buscamos contenidos en la red, lo que buscamos es un término, un concepto. Por tanto, tener una buena estrategia para cada uno de los artículos, no tan solo va a mejorar la visualización de los contenidos publicados en "grafica", sino también va a poner de relieve a los autores y a la revista.

Si bien establecer normas uniformadoras parece una limitación, en el caso del ámbito del diseño gráfico en general, y de manera específica en "grafica", se convierte en una necesidad estratégica de primer orden dado el discreto potencial de producción y visualización de los contenidos publicados. Creemos que, dada la poca tradición científica del ámbito de diseño gráfico por su juventud, el no usar palabras clave, digamos homogéneas, dispersa el potencial académico y científico de campo.

Desde nuestra perspectiva, disponer de un Theshaurus sobre los ámbitos del diseño gráfico facilitaría ese inventario de palabras que darían coherencia y potencia a los contenidos publicados. En definitiva, mayor relevancia. Ya durante el primer periodo de "Temes de Disseny" (1988-1992) la revista editada por Elisava, daba cuenta desde su "Centre de Documentació" de la elaboración de un Thesaurus específico de diseño industrial, gráfico y de interiores elaborado por el Centro (Antich: 96, p.366): "Historia del disseny gràfic, disseny gràfic, monografíes de disseny gràfic, Disseny a Catalunya i España, Moviments i Tendències, Teoria, Economia i Empresa, Marc legal, Tecnolologies i Materials, Tècniques de presentación i representació, Tipografia i Pictogrames o Informática aplicadad al disseny" quedan a la vista del siglo XXI del todo insuficientes; pero el precedente es claro: establecer una paleta de palabras clave común.

Otra alternativa de referencia bien podría ser la nomenclatura establecida por la Unesco para el campo de las ciencias y las tecnologías y que son utilizados en investigación y tesis doctorales. Los códigos Unesco indican palabras según el campo, la disciplina o la subdisciplina1.

Incluso se nos ocurre plantear la posibilidad de usar servicios públicos como lo es Termcat $^{2}$ en donde podemos encontrar palabras vinculadas a nuestra investigación, trabajo o comunicación.

Sea como sea, podemos, desde nuestra modesta intención, sugerir algunas rutinas para dar con las palabras apropiadas para un artículo científico o ensayo en "grafica".

La Primera norma seria buscar artículos de nuestro mismo tema, ámbito o campo de estudio y observar las palabras clave que utilizan. Así sa-

1. https://www.upct.es/estudios/doctorado/documentos/codigos_unesco_7809.pdf[Consulta: junio 2021]

2. (https://www.termcat.cat/ca/diccionaris-en-linia) [Consulta: junio 2021] 
bremos que nuestras palabras definen bien nuestro contenido y lo que también es importante, son específicas del objeto de estudio sobre el que desarrollamos nuestra propuesta.

La Segunda norma es pensar en qué palabras clave utilizaríamos para buscar nuestro artículo o artículos vinculados con nuestro trabajo.

La Tercera norma versa sobre el tipo y redacción de palabras, su cantidad y su posición. En este sentido, es importante seguir los criterios de la publicación. En general, las normas para editores de las publicaciones indican el número de palabras clave, siempre separadas por punto y coma. Pueden ser palabras independientes o construcciones de dos o tres palabras. La posición de las palabras es relevante. Si utilizamos palabras sueltas, la búsqueda en internet suele ser de aspectos más genéricos; en cambio, si buscamos por frases, el resultado de la búsqueda és más concreto. Primero colocaremos las más importantes y las siguientes en orden decreciente.

La Cuarta norma es no utilizar palabras que estén en el título. Ciertamente, es una cuestión recurrente. Sin embargo, dado que el título es un elemento más del conjunto de metadatos, acaba siendo redundante. En este sentido, parece más indicado utilizar sinónimos de las palabras coincidentes.

La quinta norma es no utilizar, en exceso, palabras demasiado genéricas. Así, utilizar la palabra "Diseño gráfico" en exceso para una revista especializada en diseño gráfico no parece que permita la discriminación del contenido del artículo. En general, todos los artículos publicados en "grafica" versan sobre diseño o diseño gráfico. No nos referimos solo a diseño como palabra; podríamos estar hablando de palabras genéricas como composición, historia, tipografía, etc. Por el contrario, se recomienda analizar la redacción del resumen, elemento también del conjunto de metadatos, y sintetizar las palabras o sinónimos de éstas que mejor centran nuestro tema.

La sexta norma es buscar palabras alternativas o sinónimas que nos permitan acotar y centrar nuestro tema

La séptima norma es cuidar la redacción de las palabras clave. Que estén bien escritas es fundamental. Se usará la forma oficial y reconocida del término: Un buen ejemplo de este detalle es el término Marketing, Mercadotécnica o Marqueting. También se recomienda el uso de las mayúsculas. Incluir entre las palabras clave las abreviaturas siempre que no induzcan a confusión al ser términos utilizados en diversos campos.

La octava norma consiste en probar antes de dar por definitivas las palabras clave. Así se recomienda

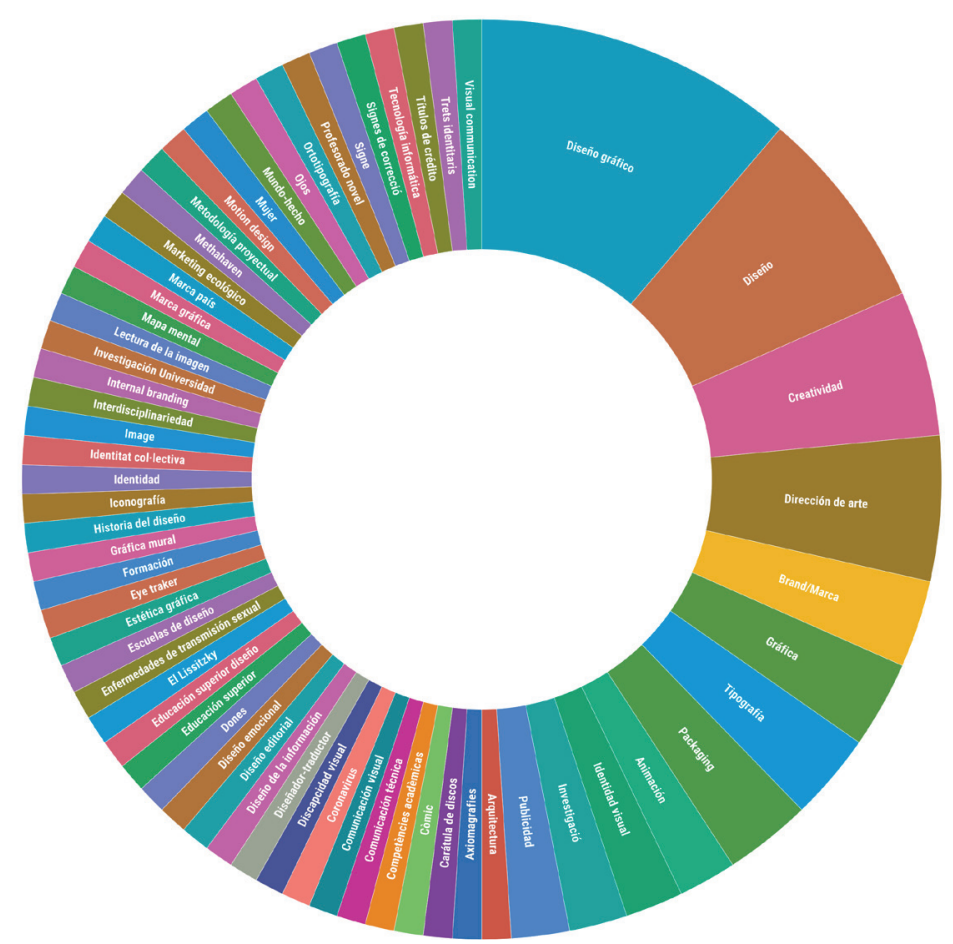

Figura 1. Representación visual del uso de las Palabras clave en "grafica" en el periodo $2012-2020$.

comprobar el rendimiento de las palabras clave en las plataformas académicas o científicas o generadores de palabras clave. Google Scholar o Google Analitics nos pueden ser de gran utilidad. Otros recursos para acertar con las palabras clave más apropiadas pueden ser: Soovle, Google Keyword Planner de AdWords, Google Correlate, o Keyword In.

Seguramente podríamos encontrar más normas pero la mayor de todas ellas es aplicar el sano juicio. Es decir, establecer una estrategia y usar la mejor táctica para conseguir nuestro objetivo. Es pertinente aquí recordar que lo importante es "hacerlo bien y hacerlo saber"; es decir: hacerlo y comunicarlo bien.

\section{Grafica y las palabras clave}

Como evidencia de esta editorial sobre las palabras clave, queremos mostrar lo ocurrido durante los últimos años en "grafica" (Fig. 1). Con tal objetivo hemos analizado las palabras clave utilizadas por los autores en los artículos sujetos a revisión doble ciego entre los años 2012 y 2020.

3. Fuente: https://www.actualidadecommerce.com/ca/6-einesde-recerca-de-paraules-clau/ [Consulta: junio 2021] 


\begin{tabular}{|c|c|c|c|}
\hline \multicolumn{4}{|l|}{ Frequencies of $\mathrm{KW} 1$} \\
\hline Levels & Counts & $\%$ of Total & Cumulative $\%$ \\
\hline Marca País & 1 & $1.0 \%$ & $1.0 \%$ \\
\hline Signe & 1 & $1.0 \%$ & $2.0 \%$ \\
\hline Creatividad & 5 & $5.1 \%$ & $7.1 \%$ \\
\hline Dones & 1 & $1.0 \%$ & $8.2 \%$ \\
\hline Ojos & 1 & $1.0 \%$ & $9.2 \%$ \\
\hline Tecnología informática & 1 & $1.0 \%$ & $10.2 \%$ \\
\hline Empaquetatge & 1 & $1.0 \%$ & $11.2 \%$ \\
\hline Envàs & 1 & $1.0 \%$ & $12.2 \%$ \\
\hline Lectura de la imagen & 1 & $1.0 \%$ & $13.3 \%$ \\
\hline Trets identitaris & 1 & $1.0 \%$ & $14.3 \%$ \\
\hline Investigació & 1 & $1.0 \%$ & $15.3 \%$ \\
\hline Identitat col-lectiva & 1 & $1.0 \%$ & $16.3 \%$ \\
\hline grafica & 2 & $2.0 \%$ & $18.4 \%$ \\
\hline Investigación & 1 & $1.0 \%$ & $19.4 \%$ \\
\hline Identidad & 1 & $1.0 \%$ & $20.4 \%$ \\
\hline Tipografía & 2 & $2.0 \%$ & $22.4 \%$ \\
\hline Mujer & 1 & $1.0 \%$ & $23.5 \%$ \\
\hline Disseny gràfic & 1 & $1.0 \%$ & $24.5 \%$ \\
\hline Arquitectura & 1 & $1.0 \%$ & $25.5 \%$ \\
\hline Comunicación técnica & 1 & $1.0 \%$ & $26.5 \%$ \\
\hline Eye traker & 1 & $1.0 \%$ & $27.6 \%$ \\
\hline Escuelas de diseño & 1 & $1.0 \%$ & $28.6 \%$ \\
\hline Publicidad & 2 & $2.0 \%$ & $30.6 \%$ \\
\hline Còmic & 1 & $1.0 \%$ & $31.6 \%$ \\
\hline Identidad visual & 2 & $2.0 \%$ & $33.7 \%$ \\
\hline Methahaven & 1 & $1.0 \%$ & $34.7 \%$ \\
\hline Enfermedades de transmisión sexsual & 1 & $1.0 \%$ & $35.7 \%$ \\
\hline Visual communication & 1 & $1.0 \%$ & $36.7 \%$ \\
\hline Diseño gráfico & 10 & $10.2 \%$ & $46.9 \%$ \\
\hline Metodología proyectual & 1 & $1.0 \%$ & $48.0 \%$ \\
\hline Interdisciplinaruedad & 1 & $1.0 \%$ & $49.0 \%$ \\
\hline Gráfica mural & 1 & $1.0 \%$ & $50.0 \%$ \\
\hline Internal branding & 1 & $1.0 \%$ & $51.0 \%$ \\
\hline Diseño editorial & 1 & $1.0 \%$ & $52.0 \%$ \\
\hline Diseñador-traductor & 1 & $1.0 \%$ & $53.1 \%$ \\
\hline Marca gráfica & 1 & $1.0 \%$ & $54.1 \%$ \\
\hline Gráfica & 1 & $1.0 \%$ & $55.1 \%$ \\
\hline Diseño & 7 & $7.1 \%$ & $62.2 \%$ \\
\hline Historia del diseño & 1 & $1.0 \%$ & $63.3 \%$ \\
\hline Competències acadèmicas & 1 & $1.0 \%$ & $64.3 \%$ \\
\hline Educación superior & 1 & $1.0 \%$ & $65.3 \%$ \\
\hline Animación & 1 & $1.0 \%$ & $66.3 \%$ \\
\hline Mapa mental & 1 & $1.0 \%$ & $67.3 \%$ \\
\hline Dirección de arte & 4 & $4.1 \%$ & $71.4 \%$ \\
\hline Tipografia & 1 & $1.0 \%$ & $72.4 \%$ \\
\hline Animació & 1 & $1.0 \%$ & $73.5 \%$ \\
\hline Comunicación visual & 1 & $1.0 \%$ & $74.5 \%$ \\
\hline Educación superior diseño & 1 & $1.0 \%$ & $75.5 \%$ \\
\hline Packaging & 1 & $1.0 \%$ & $76.5 \%$ \\
\hline Diseño de la información & 1 & $1.0 \%$ & $77.6 \%$ \\
\hline Investigación Universidad & 1 & $1.0 \%$ & $78.6 \%$ \\
\hline Signes de correcció & 1 & $1.0 \%$ & $79.6 \%$ \\
\hline Estética gráfica & 1 & $1.0 \%$ & $80.6 \%$ \\
\hline Discapcidad visual & 1 & $1.0 \%$ & $81.6 \%$ \\
\hline Image & 1 & $1.0 \%$ & $82.7 \%$ \\
\hline Profesorado novel & 1 & $1.0 \%$ & $83.7 \%$ \\
\hline Axiomagrafies & 1 & $1.0 \%$ & $84.7 \%$ \\
\hline Formación & 1 & $1.0 \%$ & $85.7 \%$ \\
\hline Mundo-hecho & 1 & $1.0 \%$ & $86.7 \%$ \\
\hline Títulos de crédito & 1 & $1.0 \%$ & $87.8 \%$ \\
\hline Motion design & 1 & $1.0 \%$ & $88.8 \%$ \\
\hline Marketing ecológico & 1 & $1.0 \%$ & $89.8 \%$ \\
\hline Diseño emocional & 1 & $1.0 \%$ & $90.8 \%$ \\
\hline Iconografía & 1 & $1.0 \%$ & $91.8 \%$ \\
\hline Coronavirus & 1 & $1.0 \%$ & $92.9 \%$ \\
\hline Carátula de discos & 1 & $1.0 \%$ & $93.9 \%$ \\
\hline Ortotipografía & 1 & $1.0 \%$ & $94.9 \%$ \\
\hline El Lissitzky & 1 & $1.0 \%$ & $95.9 \%$ \\
\hline Dirección de arte & 1 & $1.0 \%$ & $96.9 \%$ \\
\hline Brand/Marca & 3 & $3.1 \%$ & $100.0 \%$ \\
\hline
\end{tabular}

Figura 2. Frecuencia de las Palabras Clave utilizadas en "grafica" (20122020). Fuente: https://revistes.uab.cat/grafica [Consulta: junio 2021]
Quizás los resultados no sean sorprendentes, pero sí podemos incidir en dos aspectos que nos parecen importantes y que pueden hacer que pongamos nuestra atención en mejorar la elección de las palabras clave.

La primera consideración es hablar de la muestra de palabras clave utilizadas en "grafica" en el periodo 2012-2020. Así el número de palabras clave usadas por los autores nos da una muestra útil de $\mathrm{N}=98$ palabras clave utilizadas en artículos científicos y ensayos.

Queremos poner sobre la mesa es la frecuencia del uso de las palabras muestra una gran dispersión. Y por el otro, siendo "Diseño gráfico" la palabra/frase más utilizada, poco nos dice del contenido real al ser un término de carácter general. Así "Diseño gráfico" aparece un total de 10 casos, siendo la palabra clave más utilizada. Buena parte del resto de palabras clave han sido utilizadas una vez (figura 2).

La segunda consideración es el número de palabras clave por original utilizadas en los artículos (figura 3). Así, los $\mathrm{N}=98$ artículos utilizan dos palabras clave. Desde este valor se va decreciendo el número de palabras clave por artículo. De esta forma se pasa a tres palabras clave en 94 artículos, con cuatro en 82 artículos, con cinco en 51 artículos y, finalmente, hay 11 artículos con seis palabras clave.

No podemos dar soluciones mágicas, pero sí poner de relieve que los metadatos de los artículos son un elemento fundamental para la transmisión de conocimiento. Uno de los elementos más relevantes de esos metadatos son las palabras clave. Sería de desear que, en adelante, todos focalicemos esas palabras más apropiadas para nuestros artículos.

\section{Grafica V9 \#18}

Es el momento de introducir los artículos que publicamos en el presente número de "grafica". Son tres artículos de investigación y cuatro ensayos que están acompañados por el Expertia de Javier Solas. Éste versa sobre las tres primeras convocatorias de los Premios "Gaudeamus Projecta" (2017, 2018 y 2019) que otorga el Col-legi Oficial de Disseny Gràfic de Catalunya que reconocen y premian la excelencia académica de los proyectos de fin de grado en diseño gráfico presentados.

Por su parte, las siete aportaciones entre artículos científicos (3) y ensayos (4), han seguido el proceso de revisión ciega de doble pares que sigue "grafica" para la evaluación de los originales y queremos desde aquí agradecer a los revisores, el trabajo poco visible pero que redunda en la calidad de la revista grafica. Ya anunciábamos en el ante- 
rior número la incorporación de "grafica" al listado de las revistas científicas Scopus. En este queremos señalar también que la revista ha optado por el sello de calidad editorial Fecyt para las revista científicas y por el momento, se ha superado la primera fase; veremos la evaluación otorgada en la segunda y definitiva fase. Sea cual sea el resultado final, esto nos lleva a pensar que el trabajo que realizamos, editores, revisores, autores y naturalmente, también lectores, va en la buena dirección. Solo podemos reiterar una vez más desde estas líneas: nuestras felicitaciones a todos.

El primer artículo de investigación es "¿Qué papel juega el diseño gráfico en la accesibilidad web?" escrito por Rubén Alcaraz-Martínez de la Universitat de Barcelona, y Lluc Massaguer de la Universitat Oberta de Catalunya. El objetivo de la aportación es analizar la importancia del diseño gráfico en la creación de un sitio web accesible conforme a las WCAG del W3C. Los resultados muestran la importancia, en este sentido, del perfil del diseñador/a gráfico.

El segundo artículo de investigación corresponde al texto "El impacto del flat design en el reconocimiento de marcas automovilísticas" firmado por Gema Bonales-Daimiel, Luis Mañas-Viniegra e Isidro Jiménez-Gómez, todos ellos de la Universidad Complutense de Madrid. El artículo presenta la tendencia que nace del diseño web y que combina funcionalidad y estética, simplificando formas y colores para favorecer el recuerdo. La investigación analiza la percepción cognitiva que los jóvenes tienen hacia las marcas automovilísticas en términos de recuerdo y representación y el papel que el diseño plano desempeña. Los resultados corroboran que las marcas gráficas más representadas fueron las más sencillas desde el punto de vista gráfico.

En cuanto al tercer artículo de investigación, "El tratamiento formal de los actos terroristas en la prensa generalista española. El atentado de Barcelona de 2017", firmado por Sara Aranda Martínez presenta, a través de la metodología del análisis de contenido, una aproximación al tratamiento formal que los diarios El País, El Mundo, La Vanguardia y El Periódico dieron del atentado acaecido en Barcelona el 17 de agosto de 2017. Los resultados dan cuenta de las estrategias formales seguidas.

Estos tres artículos de investigación estan acompañados por el texto de la docente investigadora de la Corporación Universitaria de Asturias, Margarita Gutiérrez, "El Marketing, el Greenwashing y su influencia en el Brand Equity"; por otro ensayo, en este caso de Pilar Vicente e Isidoro Arroyo-Almaraz, de la Universidad Rey Juan Carlos, Madrid (España), "La comunicación gráfi-

\begin{tabular}{ccccccc}
\hline & KW1 & KW2 & KW3 & KW4 & KW5 & KW6 \\
\hline $\mathrm{N}$ & 98 & 98 & 94 & 82 & 51 & 11 \\
\hline
\end{tabular}

Figura 3. Número de palabras clave en artículos publicados en grafica en el periodo 2012-2020. Fuente: https://revistes.uab.cat/grafica [Consulta: junio 2021]

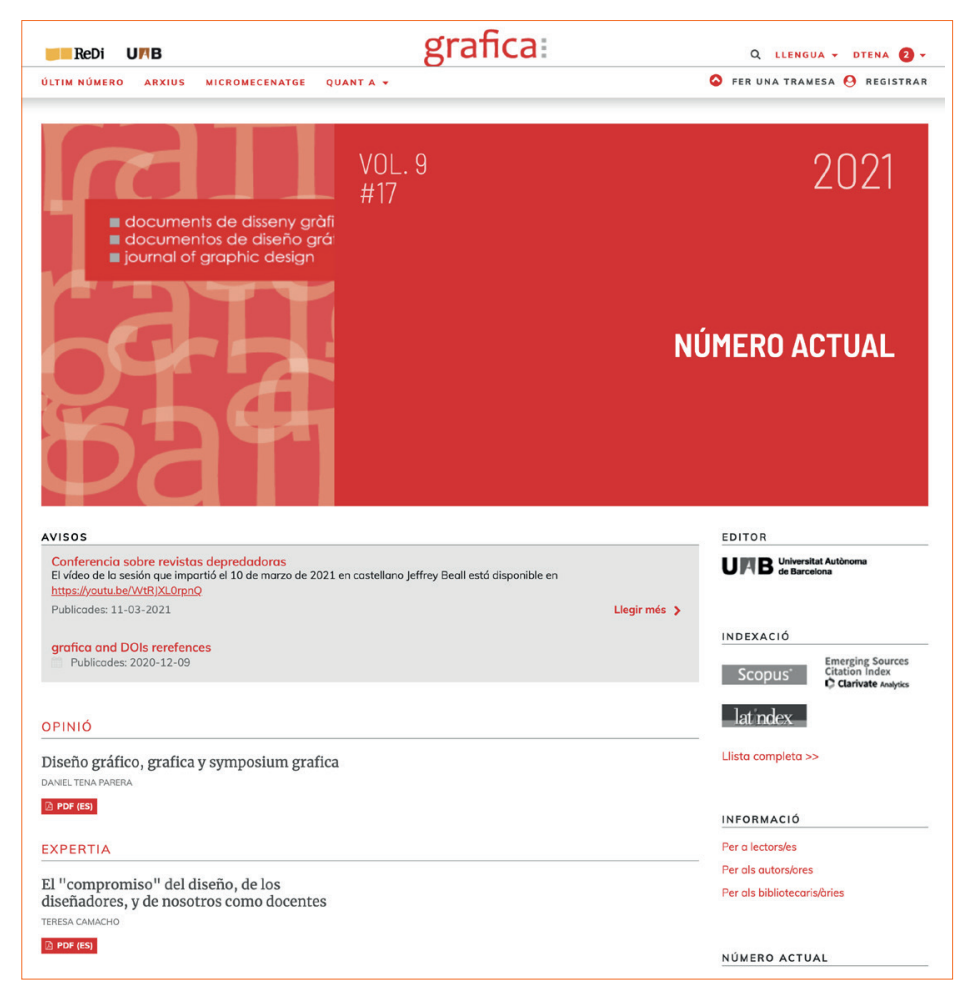

Figura 4. Aspecto de grafica en OJS3. Fuente: https://revistes.uab.cat/grafica [Consulta: junio 2021]

ca en la prensa femenina: estudio de las portadas de la revista Telva (2011-2020)"; por el también ensayo, "El arte y la ornamentación en los objetos y los espacios construidos" de Rosario Vicente y Joan Sanz, ambos de la Universidad de Granada; y finalmente, presentamos el ensayo de Enrique López de la Universidad de Granada, "Evolución de la identidad institucional en Andalucía".

Solo nos queda apuntar una última nota al pie de pàgina: con este número, la plataforma digital de "grafica" ha pasado de la versión OJS2 a la versión OJS3 con lo que ha cambiado su apariencia visual y sus procedimientos técnicos. Esperamos que sea de mayor ayuda (figura 4).

Buena y beneficiosa lectura. 
Referencias bibliográficas

Antich, Berta. "The Elisava Document Centre". Temes de Disseny, [online], 1996, Num. 13, pp. 366-71, https://raco.cat/index.php/Temes/article/view/359343 [View: 21-06-2021].

https://revistes.uab.cat/grafica [Consulta: junio 2021]

https://www.actualidadecommerce.com/ca/6eines-de-recerca-de-paraules-clau/ [Consulta: junio 2021] https://www.termcat.cat/ca/diccionaris-en-linia [Consulta: junio 2021]

https://www.actualidadecommerce.com/ca/6eines-de-recerca-de-paraules-clau/ [Consulta: junio 2021]

https://www.researchvoyage.com/top-10-rulesto-identify-keywords-for-your-research-paper/\# [Consulta: junio 2021]

https://www.upct.es/estudios/doctorado/documentos/codigos_unesco_7809.pdf [Consulta: junio 2021] 